\title{
Differential scanning calorimetry of plasma in glioblastoma: toward a new prognostic / monitoring tool
}

\author{
Philipp O. Tsvetkovi, Emeline Tabouret, ${ }^{1,2}$, Andrei Y. Roman ${ }^{1,5}$, Sylvie Romain ${ }^{3}$, \\ Céline Bequet ${ }^{2}$, Olga Ishimbaeva ${ }^{1}$, Stéphane Honoré ${ }^{1}$, Dominique Figarella- \\ Branger $^{4}$, Olivier Chinot $^{1,2}$ and François Devred ${ }^{1}$ \\ ${ }^{1}$ Aix-Marseille Université, CNRS, INP, Inst Neurophysiopathol, Faculté de Pharmacie de Marseille, 13385 Marseille, France \\ ${ }^{2}$ AP-HM, Hôpital de la Timone, Service de Neuro-Oncologie, 13005 Marseille, France \\ ${ }^{3}$ AP-HM, Faculté de Médecine Nord, Service de Transfert d'Oncologie Biologique, 13015 Marseille, France \\ ${ }^{4}$ AP-HM, Hôpital de la Timone, Service d'Anatomopathologie, 13005 Marseille, France \\ ${ }^{5}$ Institute of Physiologically Active Compounds, RAS, 142432 Chernogolovka, Russian Federation \\ Correspondence to: François Devred, email: francois.devred@univ-amu.fr \\ Keywords: glioblastoma; differential scanning calorimetry; disease monitoring; plasma; calorimetric signature \\ Received: August 20, $2017 \quad$ Accepted: January 09, $2018 \quad$ Published: January 25, 2018 \\ Copyright: Tsvetkov et al. This is an open-access article distributed under the terms of the Creative Commons Attribution License \\ 3.0 (CC BY 3.0), which permits unrestricted use, distribution, and reproduction in any medium, provided the original author and \\ source are credited.
}

\section{ABSTRACT}

Glioblastoma is the most frequent and aggressive primary brain tumor in adults. Recently, a growing number of studies have shown that denaturation profile of plasma samples obtained by differential scanning calorimetry (DSC) can represent a signature of a disease. In this study, we analyzed for the first time the DSC denaturation profiles of the plasma from patients with recurrent glioblastoma $(n=17)$. Comparison to the one of healthy individuals $(n=10)$ and to already described profiles in others cancer showed clear differences suggesting that this DSC profile may constitute a signature of glioblastoma. Parameters extracted from these profiles were used for cluster analysis which revealed the existence of glioblastoma profile subgroups which correlated with prognostic factors. Moreover, we showed that the presence of circulating bevacizumab and carmustine did not alter this calorimetric signature of the disease, indicating that an evolution of the profile could be followed without being masked by ongoing systemic treatment. Thus, our results constitute a very promising proof of principle that a specific calorimetric profile could be detected in the plasma of glioblastoma patients. Moreover, we believe that our findings point to a potential easy-to-use noninvasive monitoring tool for glioblastoma patients.

\section{INTRODUCTION}

Glioblastoma $(\mathrm{GB})$ is the most frequent and aggressive primary brain tumor in adults. It is characterized by cellular atypia, severe necrosis, and high rate of angiogenesis [1]. To date, response after standard first line treatment associating radiotherapy and temozolomide is very heterogeneous, leading to inevitable recurrence [2]. At relapse, few treatment options are available and remain associated with heterogeneous response rates [3]. In this indication, the use of bevacizumab is associated with favorable response rate and improved progressionfree survival $[4,5]$. In this context of limited systemic therapies, accurate and timely detection of the disease recurrence is crucial in order to optimize the therapeutic options and to improve the patient management. To date, response assessment has been based on neuro-imaging (MRI), steroid dose and clinical examination, which can be difficult to interpret, especially following radiotherapy, anti-angiogenic therapy or immunotherapy $[6,7]$. Thus, the identification of a new easy-to-use method to monitor glioblastoma evolution is an urgent need in the neurooncology field. 
Recently, it was suggested that differential scanning calorimetry (DSC), a biophysical method routinely used to measure temperature-induced denaturation of purified proteins [8, 9], could have a new disease-monitoring application. For purified single-domain proteins, the profile of denaturation obtained by DSC is usually characterized by a single peak with its surface corresponding to the enthalpy of protein denaturation $(\Delta \mathrm{H})$, that is additional energy that should be applied to the protein solution to reach full protein unfolding [10]. The maximum of this peak corresponds to the melting temperature (Tm). These two parameters are characteristic for each protein. They can be altered by protein modification, such as mutations or post-translational modifications [8], or by interaction with its binding partners, such as other proteins, small molecules and even ions [911]. It has been recently shown that applying DSC directly to biofluids, such as serum, plasma or cerebro-spinal fluid (CSF), resulted in reproducible specific signature profiles characteristic of the clinical state of an individual [12]. With such complex biological samples, the obtained denaturation profile corresponds to the superposition of denaturation calorimetric profiles of all components in the sample. The modifications in blood composition of patients suffering from cancer can lead to significant changes in calorimetric profile of plasma. Over the past five years, an increasing number of publications have shown that such impact exists in many diseases including diabetes, Lyme disease, as well as several types of cancer [12]. For example, calorimetric profile of plasma samples from patients affected by cervical, endometrial, ovarian or lung cancers, was different from that of healthy controls $[13,14]$. In the later studies, the difference between profile of healthy controls and patients has also been observed for breast, multiple myeloma, colorectal and gastric cancer [15-17]. Moreover, superposition of DSC profiles obtained from these cancers suggested that each type of cancer may have a characteristic thermogram [14]. Finally, a preliminary study conducted on the CSF of six GB patients revealed a specific profile suggesting the existence of a biofluid signature of GB [18].

Recurrent GB is generally associated with a certain tumor burden, increasing the chance to detect potential circulating markers. Our primary objective was to analyze the DSC profile of plasma of recurrent GB patients. Our secondary objectives were (i) to correlate this profile to patient characteristics, (ii) to evaluate its potential prognostic value and (iii) to analyze the impact of circulating bevacizumab and carmustine agents on DSC profile.

\section{RESULTS}

\section{Patient characteristics (Table 1)}

Patient cohort was composed of 17 GB patients with a median age of 62.5 years (range, 42-89) and a median Karnofsky Performance Status (KPS) of 70 (range, 60-90) at relapse. For the majority of patients, the first line treatment consisted of surgical resection followed by radio-chemotherapy. At relapse, median diameter of enhanced lesion(s) was 45mm (range, 20-80). The control cohort used in this study was composed of 2 women and, 8 men, with a median age of 36.7 years (range, 24-51) and a body mass index of 23.3 (range, 19.2-30).

\section{DSC profiling of plasma samples}

Plasma samples from 10 healthy individuals and 17 GB patients were analyzed by DSC to obtain denaturation profiles. Average profiles of healthy individuals and GB patients are shown in the Figure 1A. Plasma denaturation profiles of healthy individuals are homogeneous and are characterized by a main maximum at $63^{\circ} \mathrm{C}$ and smaller one at $70^{\circ} \mathrm{C}$ as previously described [12]. Profiles of GB patients are characterized by a negative peak around $80^{\circ} \mathrm{C}$ and a less pronounced difference between maxima at $63^{\circ} \mathrm{C}$ and $70^{\circ} \mathrm{C}$. The difference between healthy individuals and GB profiles is significant in the temperature ranges 60$65^{\circ} \mathrm{C}$ and $75-85^{\circ} \mathrm{C}(\mathrm{p}<0.001)$ (Figure 1B).

\section{Correlation of DSC parameters with patient characteristics and survival}

In order to evaluate the potential correlation between patient characteristics and DSC profile, minimal and maximal curve values, associated temperatures, and areas under the curve were extracted from the individual profiles obtained from GB patients. None of these parameters were correlated to classical patient prognostic factors (age, steroid dose, KPS) or other patient characteristics (gender, body mass index). Moreover, DSC features were not correlated to the neuro-imaging characteristics of patients (maximal diameter of enhanced lesion) or to the trough concentration of bevacizumab at D21. Remarkably, several of DSC parameters turned out to have a prognostic value. Indeed, the temperatures of minimal $(p=0.035)$ and maximal $(p=0.014)$ curve values were associated with progression-free survival (Figure $2 \mathrm{~A}$ and $2 \mathrm{~B}$ ), while the area under the curve was significantly associated with overall survival ( $p=0.025$ ) (Figure $2 \mathrm{C}$ and 2D).

Despite of our limited number of samples, in an exploratory perspective, we conducted a cluster analysis in order to take into account more DSC profile parameters (see Methods) in a comprehensive way and to perform a finer statistical analysis of the thermograms. We have applied principal components analysis followed by several clustering methods and distance functions most of which clearly separated healthy from GB profiles. For example, as shown in Figure 3, analysis using "Optimize" method with Euclidian distance function showed the existence of two distinct groups of signatures $(\mathrm{p}<0.005)$ corresponding to healthy controls (triangles) and GB patients (circles). Moreover, using this method we were also able to identify 3 clusters within the GB group (different colors) which 
Table 1: Patient characteristics

\begin{tabular}{|c|c|c|}
\hline Characteristics & $\mathbf{N}$ & $\%$ \\
\hline \multicolumn{3}{|l|}{ Gender } \\
\hline Women & 5 & 30 \\
\hline Men & 12 & 70 \\
\hline \multicolumn{3}{|l|}{ Type of surgery } \\
\hline Gross total resection & 10 & 59 \\
\hline Partial resection & 7 & 41 \\
\hline \multicolumn{3}{|l|}{ Histology } \\
\hline IDHwt glioblastoma & 17 & 100 \\
\hline \multicolumn{3}{|l|}{ MGMT promoter } \\
\hline Methylated & 1 & 11 \\
\hline Unmethylated & 8 & 89 \\
\hline \multicolumn{3}{|l|}{ First line treatment } \\
\hline Radiotherapy alone & 1 & 6 \\
\hline Temozolomide alone & 1 & 6 \\
\hline Stupp protocol & 15 & 88 \\
\hline \multicolumn{3}{|l|}{ Before bevacizumab $+\mathrm{BCNU}$ treatment } \\
\hline Age (years: median, range) & \multicolumn{2}{|c|}{$62,5(42,5-89,8)$} \\
\hline Body Mass Index (median, range) & \multicolumn{2}{|c|}{$24,1(18,1-32,1)$} \\
\hline Delay between initial diagnosis and relapse (months: median, range) & \multicolumn{2}{|c|}{$13,3(4,1-64,7)$} \\
\hline Karnofsky Performans Status (median) & \multicolumn{2}{|c|}{70} \\
\hline 60 & 3 & 19 \\
\hline 70 & 6 & 37 \\
\hline 80 & 3 & 19 \\
\hline 90 & 4 & 25 \\
\hline Patients under steroid & 8 & 47 \\
\hline Tumor diameter* ( $\mathrm{mm}$ : median, range) & \multicolumn{2}{|c|}{$45(20-80)$} \\
\hline
\end{tabular}

Wt : wild-type

* Diameter of contrast enhancement

were significantly different from each other in terms of Progression Free Survival $(p<0.005)$ and overall survival $(p=0.03)$. Using Manhattan distance function gave identical clusters.

Finally, we analyzed the association between clusters or DSC features with the time between the end of radiotherapy and relapse and found no correlation.

\section{Impact of treatment on DSC profile}

To test the impact of a systemic pharmacologic treatment on the denaturation profile, we compared plasma samples before and 21 days after treatment with bevacizumab and carmustine (Figure 4A). No significant modifications of DSC parameters were observed between the first (D1) and the second calorimetric profile (D21), suggesting that remaining circulating bevacizumab and carmustine in plasma do not interfere with the GB calorimetric profile (Figure 4B). When the patients were stratified according to the objective response (responders versus non responders), no significant difference was observed at D21 in the neither subgroups.

\section{DISCUSSION}

DSC has been previously used to detect specific biofluid denaturation profiles in autoimmune and infectious diseases, chronic health conditions as well as in a growing range of cancers [12]. In this report, we show for the first time the existence of a specific plasma denaturation profile of patients with recurrent GB which differs from the profile of healthy individuals and from all previously published disease profiles. The presence of such profile in plasma of GB patients thus opens up 
the path for a potential new method for monitoring of GB. DSC profiles were previously analyzed in the cerebrospinal fluid of six GB patients showing interesting but limited results [18]. In another study, this technique has been applied directly to the brain tissue showing differences between distinct stages of gliomas as well as control brain tissue [19]. However, this technique is limited by the amount of tissue required and by the access to the tumor. In our study, such a signature was observed in plasma samples. Since drawing blood is less invasive compared to lumbar puncture, this method would be much easier to implement for daily practice in clinics, making it an effective and convenient tool for disease monitoring. Indeed, sample analysis by DSC is a wellestablished method that is highly reproducible, relatively inexpensive, demands simple processing and requires little plasma material. Thus, plasma denaturation signatures could represent a valuable addition to the current tools that monitor the disease and guide clinical GB management.
In our study, we have also shown that these profiles can be analyzed automatically using classification methods and that this classification unambiguously separated profiles between healthy and GB. In an exploratory approach, we also found clustering settings that revealed subgroups in the GB population with different prognostic values. The DSC profile appears to be related to prognosis independently of the classical patient prognostic factors, such as age and KPS or other patient characteristics such as gender, body mass index. This prognostic value might be helpful during the course of the disease, although considering our small number of samples it will need to be confirmed in a larger cohort and to be tested also in the setting of the initial GB diagnosis. Interestingly, we did not observe significant differences between profiles obtained before and after bevacizumab treatment. Since second plasma sample was collected only three weeks after the beginning of treatment, it was probably too early to observe any correlation between an evolution of the

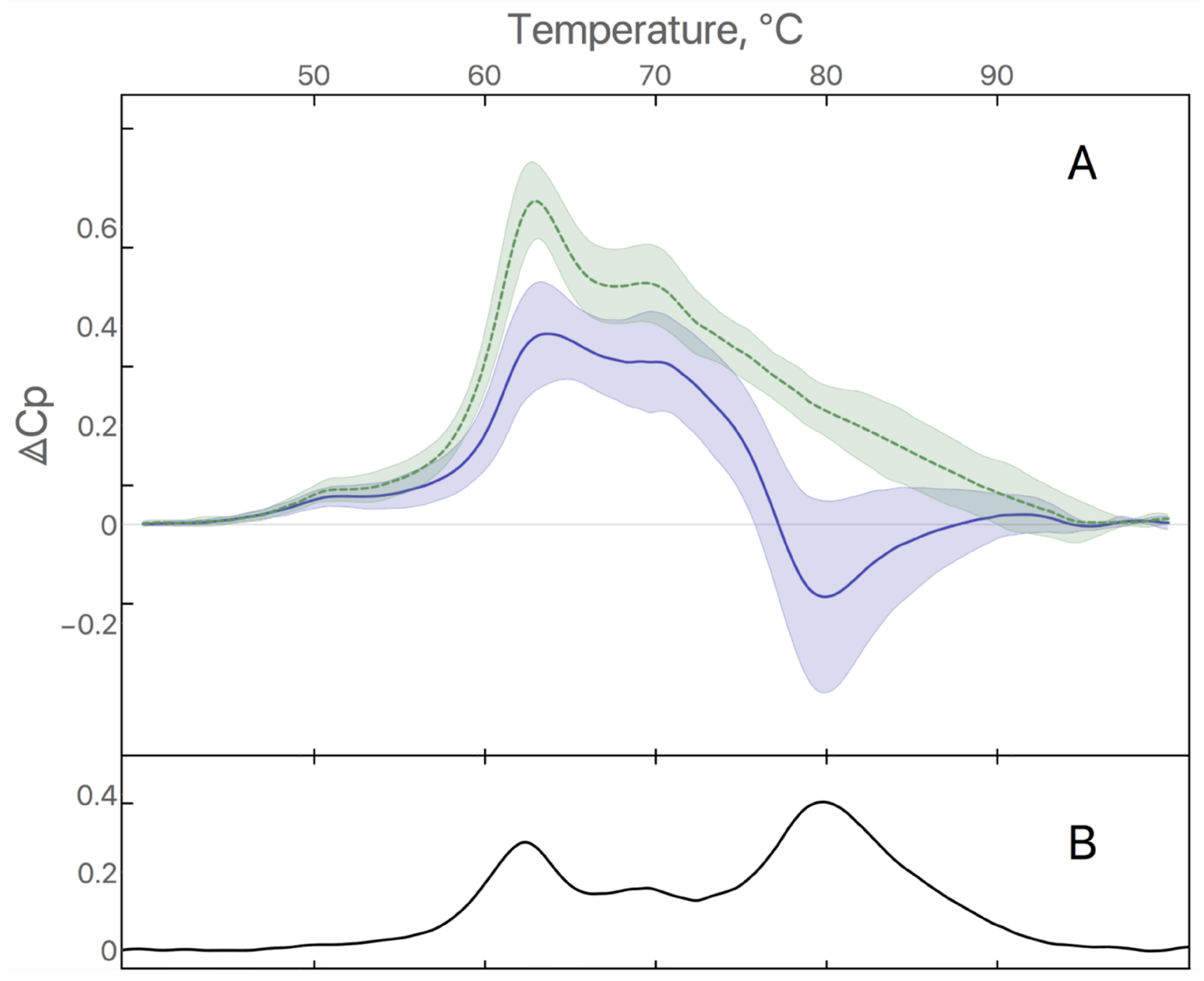

Figure 1: (A) Average of plasma denaturation profiles from 10 healthy individuals (green dashed curve) and 17 GB patients (blue solid curve). Filled area corresponds to standard deviation. (B) Difference between average of plasma denaturation profiles from healthy individuals and GB patients. 
A

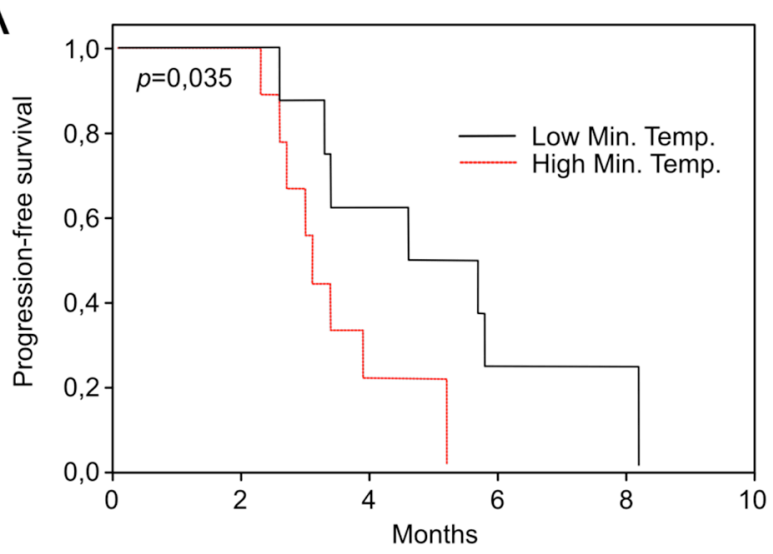

C

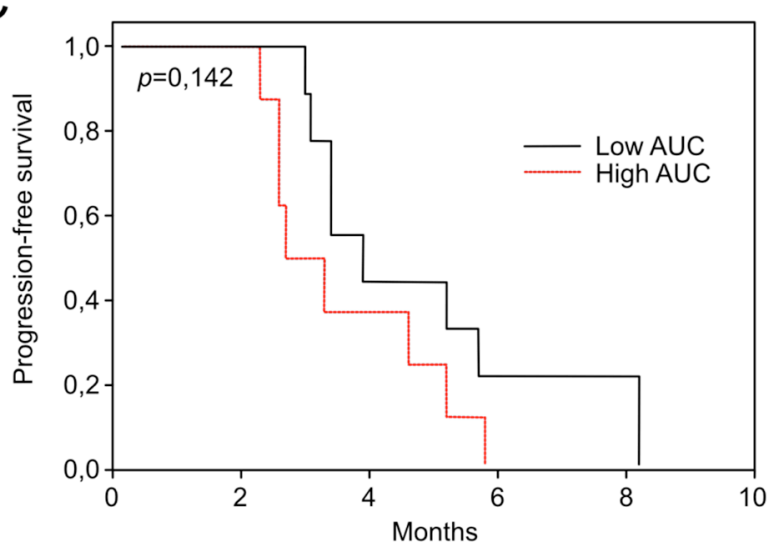

B

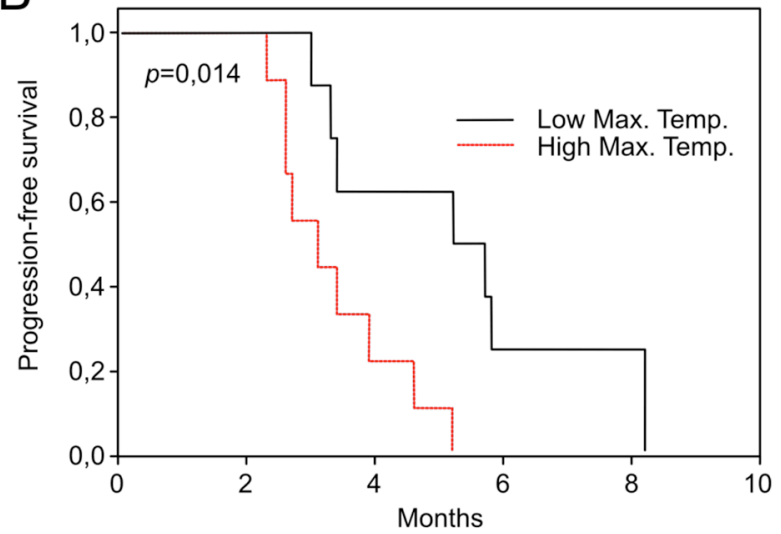

$\mathrm{D}$

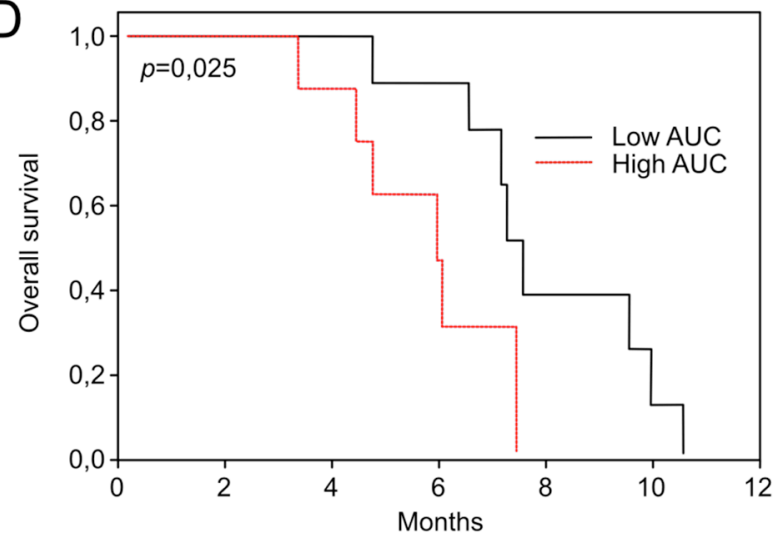

Figure 2: Progression-free survival according to the temperature of minimal (A) and maximal (B) values of denaturation profiles of GB patients, dichotomized by the median value. Progression-Free survival (C) and overall survival (D) according the Area Under the Curve (AUC) values of denaturation profiles of GB patients, dichotomized by the median value.

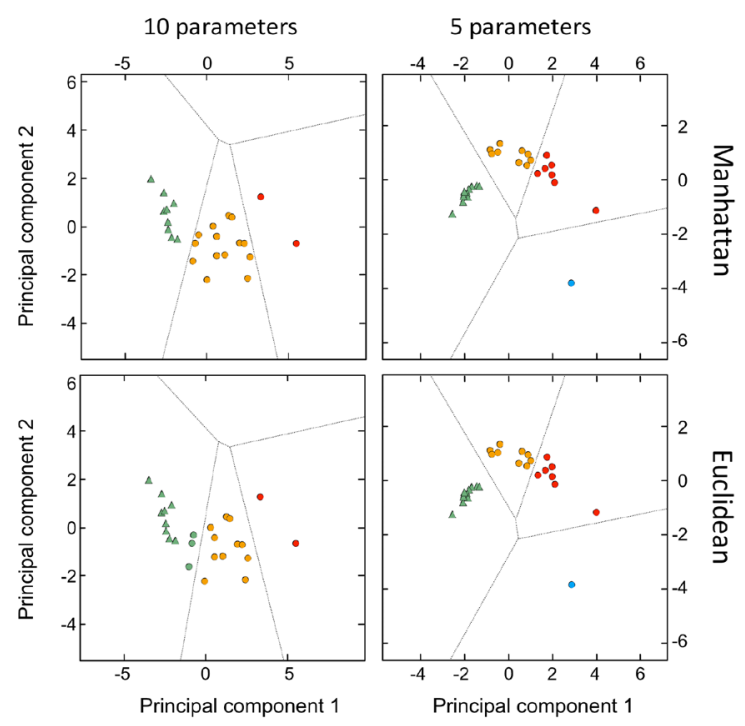

Figure 3: Principal component analysis (PCA)-based clustering by local optimization. The two major principal components explain $88 \%$ of the total variance plotted for the 10 healthy individuals and 17 GB patients. The different groups are indicated by different colors (triangles for healthy individuals and circles for GB patients). 
DSC profile and disease response. Nevertheless in this context, our results suggest that the presence of circulating drugs, such as bevacizumab, does not alter the calorimetric signature of the disease, indicating that an evolution of the profile could be followed without being masked by ongoing treatment. For all these reasons, our findings constitute a promising proof of principle that DSC could be used for a better management of GB patients.

In addition to the potential clinical application of DSC, it can also be used to improve our understanding of the mechanism of GB development. Indeed, DSC denaturation profile of a complex solution such as plasma corresponds to superposition of denaturation calorimetric profiles of all components in the plasma. In practice, the overall profile corresponds to the denaturation of the most abundant proteins, which are albumin, immunoglobulin $\mathrm{G}$, fibrinogen, immunoglobuline A, alpha2macroglobulin, haptoglobulin and alphalantitrypsin [12, 14]. Blood homeostasis maintains the concentration of these proteins constant in any healthy organism, resulting in a reproducible "healthy" denaturation signature. A modification of the profile is a sign of a change in the homeostasis which can have several causes: a change in the concentration of the most abundant proteins, any posttranslation chemical modification of one of them or the binding of a ligand, constituting a potential biomarker. Thus, this method could reveal the presence of biomarkers in the plasma directly by their impact on the calorimetric profile.

Despite several limitations, such as the limited number of samples and the monocentric nature of our study, we believe that this study constitutes the proof of principle that DSC could be used as a new approach for prognostic evaluation and/or disease monitoring with minimal handling of plasma samples. Even though our results are statistically significant, they should be confirmed in a larger population to evaluate the robustness of our approach. It would also be interesting to analyze the DSC profile of patients' plasma sample at initial diagnosis and during the treatment in a larger prospective cohort,

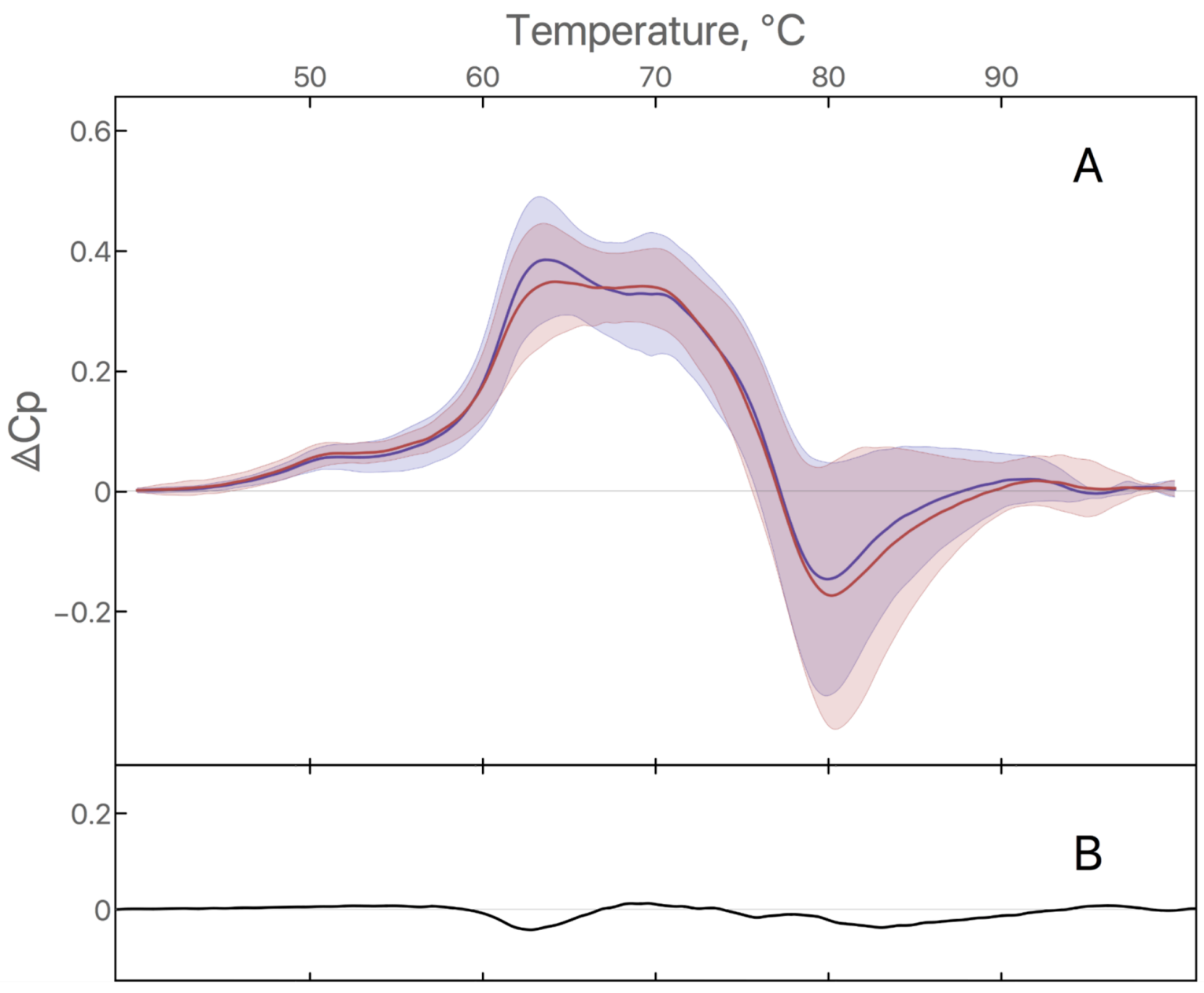

Figure 4: (A) DSC signature of plasma from GB patients at D1 and D21: Average of plasma denaturation profiles from 17 GB patients at D1 (blue curve) and the same 17 GB patients at D21 (red curve). Filled area corresponds to standard deviation. (B) Difference between average of plasma denaturation profiles of GB patients at D21 and D1. 
including other forms of gliomas. It will help to identify potential specific variations of DSC profile according to grading, prognostic and/or treatment, and thus address the ability of DSC as a new marker of glioblastoma.

\section{MATERIALS AND METHODS}

\section{Patients}

We retrospectively included all adult patients referred to our institution for recurrent $I D H$ wild-type glioblastoma who received bevacizumab at the dose of 15 $\mathrm{mg} / \mathrm{kg}$ every 3 weeks in association with carmustine at the dose of $150 \mathrm{mg} / \mathrm{m}^{2}$ every 6 weeks for whom plasma samples were available (Assistance Publique-Hôpitaux de Marseille Tumor Bank, authorization number 2013-1786) before bevacizumab administration (D1) and at the time of the second bevacizumab administration (D21). Clinical and imaging evaluations were performed every 3 weeks and 6 weeks respectively. Treatment responses and disease progression were reviewed using the RANO criteria [20]. All patients provided written informed consent in accordance with institutional, national guidelines and the Declaration of Helsinki.

\section{Plasma samples}

Plasma samples were collected at baseline, before bevacizumab administration (D1), and before the second bevacizumab administration (D21). Clinical data and neuro-imaging characteristics (when available) were recorded and the bevacizumab trough concentration was determined before the second administration. Plasmas from 10 healthy controls were also collected and processed in an identical way to the patients' samples (blood drawn in the hospital, plasma stored in the same biobank) before being analyzed. Plasma concentration of bevacizumab was quantified by enzyme linked immunosorbent assay (Elisa, Theradiag, Croissy Beaubourg, France) [21].

\section{Sample preparation}

Unprocessed plasma samples were stored at $-80^{\circ} \mathrm{C}$. No other specific purification step was added in order not to perturb the interactome or alter the chemical state of plasma proteins. Before DSC analysis, samples were thawed rapidly at $37^{\circ} \mathrm{C}$ and then diluted 25 times into the final volume of $1 \mathrm{~mL}$ of phosphate-buffered saline (PBS) buffer with sodium citrate $13 \mathrm{mM}$. Diluted samples were stored at $-20^{\circ} \mathrm{C}$ when not in use.

\section{Sample analysis by DSC}

DSC thermograms were collected using a highsensitivity differential scanning VP-DSC microcalorimeter (MicroCal, Northampton, MA, now part of Malvern Instruments Ltd) according to the manufacturer's instructions. $580 \mu \mathrm{L}$ of sample were used to fill the DSC chamber. Scans were recorded from $10^{\circ} \mathrm{C}$ to $110^{\circ} \mathrm{C}$ with a ramp heating rate of $1^{\circ} \mathrm{C} / \mathrm{min}$ using the midfeedback mode and a filtering period of $2 \mathrm{~s}$. Thermograms were treated using software Origin 7 (OriginLab Corporation, Northampton, MA) as previously described [22]. After the first run was completed, a second run of the same sample was performed without emptying the cell and subtracted from the raw data obtained in the first scan. Thermograms were plotted as the excess partial molar heat capacity (cal $\mathrm{K}^{-1} \mathrm{~mol}^{-1}$ ) versus temperature $\left({ }^{\circ} \mathrm{C}\right)$. Global protein concentration was not normalized so that the profiles obtained reflected the possible differences in protein content of plasma.

\section{Statistical analyses}

Categorical variables were presented as frequencies and percentages, continuous variables as median and range. For survival analyses, continuous variables were dichotomized by the median value. Overall survival (OS) was defined to be the time from first bevacizumab administration to death from any cause, censored at the date of last contact. ProgressionFree Survival (PFS) was the time from first bevacizumab administration to documented progression or death, censored at the date of the last documented disease evaluation. KaplanMeier method was used to estimate survivals distributions. Log-rank tests were used for univariate comparisons. MannWhitney U-test was used to compare quantitative and qualitative values; qualitative values were analyzed by Fisher exact test and Chi 2 test. Correlations were analyzed using the Spearman test. All reported $p$-values were two-sided, and $p<0.05$ was considered statistically significant. Minimal and maximal curve values, associated temperatures, and areas under the curve were then extracted from the denaturation profiles to be compared directly between patients and controls. All statistical analyses were performed by PASW Statistics 22 from IBM Corporation.

To explore the DSC profiles by principal component and clustering analysis, we used either 10 or the 5 following profile parameters: heat capacity (cal $\mathrm{K}^{-1} \mathrm{~mol}^{-1}$ ) and temperature $\left({ }^{\circ} \mathrm{C}\right)$ of the negative peak; the minimal and the maximal values of the heat capacity as well as the temperature where the heat capacity is minimal. Standardization (z-score) and cluster analysis were performed in Mathematica 10 software from Wolfram Research using the following methods: "Agglomerate" which finds clustering hierarchically, "Optimize" which finds clustering by local optimization, "KMeans" which uses k-means clustering algorithm, "KMedoids" which partitions around medoids and "Spectral" which uses spectral clustering algorithm, with Manhattan or Euclidian distances.

\section{CONCLUSION}

In conclusion, we describe a novel method which presents the advantage of revealing the presence of 
markers in the plasma directly by their impact on the DSC denaturation profile. This approach not only opens the path for rational search of new biomarkers, but also more importantly opens up the perspective of a new easy-touse non-invasive monitoring tool for GB patients. A larger study will now be needed to confirm the specificity of the signature and the extent of its prognostic value and the way its profile changes during treatment.

\section{Author contributions}

P.O.T. designed the research and supervised data analysis; E. T. supervised the plasma collection, performed the statistical analysis and wrote the manuscript; A.Yu.R performed DSC experiments and treated experimental curves; S.R. and C.B. collected patient samples; O. I. performed cluster analysis; S. H. and D.F.B. supervised the research; O. C. supervised the plasma collection; F. D. designed and supervised the research and wrote the manuscript. All authors critically reviewed iterations of the manuscript and approved the final draft for submission.

\section{ACKNOWLEDGMENTS}

Plasma from patients belonging to the Marseille Glioma cohort (CPP number: 2014-A00585-42) were retrieved from the AP-HM tumor bank (AC-2013-1786 / CRB number BB-0033-00097). Thanks to Svetlana Gorokhova for helpful discussions.

\section{CONFLICTS OF INTEREST}

The Authors declare no conflicts of interest.

\section{FUNDING}

This work has been supported by the SIRICMarseille Glioma program (Grant INCa-DGOS-Inserm 6038), by the Fondation ARC pour la recherche sur le cancer, by research funding from the Cancéropôle PACA and INCA, GEFLUC Marseille and by the patient association ARTC-Sud.

\section{REFERENCES}

1. Louis DN, Perry A, Reifenberger G, von Deimling A, Figarella-Branger D, Cavenee WK, Ohgaki H, Wiestler OD, Kleihues P, Ellison DW. The 2016 World Health Organization classification of tumors of the central nervous system: a summary. Acta Neuropathol (Berl). 2016; 131:803-20. https://doi.org/10.1007/s00401-016-1545-1.

2. Stupp R, Mason WP, van den Bent MJ, Weller M, Fisher B, Taphoorn MJB, Belanger K, Brandes AA, Marosi C, Bogdahn U, Curschmann J, Janzer RC, Ludwin SK, et al. Radiotherapy plus concomitant and adjuvant temozolomide for glioblastoma. N Engl J Med. 2005; 352:987-96. https:// doi.org/10.1056/NEJMoa043330.

3. Alexander BM, Cloughesy TF. Adult glioblastoma. J Clin Oncol. 2017; 35:2402-9. https://doi.org/10.1200/ JCO.2017.73.0119.

4. Friedman HS, Prados MD, Wen PY, Mikkelsen T, Schiff D, Abrey LE, Yung WKA, Paleologos N, Nicholas MK, Jensen R, Vredenburgh J, Huang J, Zheng M, et al. Bevacizumab alone and in combination with irinotecan in recurrent glioblastoma. J Clin Oncol. 2009; 27:4733-40. https://doi. org/10.1200/JCO.2008.19.8721.

5. Tabouret E, Barrie M, Thiebaut A, Matta M, Boucard C, Autran D, Loundou A, Chinot O. Limited impact of prognostic factors in patients with recurrent glioblastoma multiforme treated with a bevacizumab-based regimen. J Neurooncol. 2013; 114:191-8. https://doi.org/10.1007/ s11060-013-1170-y.

6. Ellingson BM, Chung C, Pope WB, Boxerman JL, Kaufmann TJ. Pseudoprogression, radionecrosis, inflammation or true tumor progression? challenges associated with glioblastoma response assessment in an evolving therapeutic landscape. J Neurooncol. 2017; 134:495-504. https://doi.org/10.1007/ s11060-017-2375-2.

7. Wen PY, Macdonald DR, Reardon DA, Cloughesy TF, Sorensen AG, Galanis E, Degroot J, Wick W, Gilbert MR, Lassman AB, Tsien C, Mikkelsen T, Wong ET, et al. Updated response assessment criteria for high-grade gliomas: response assessment in neuro-oncology working group. J Clin Oncol. 2010; 28:1963-72. https://doi. org/10.1200/JCO.2009.26.3541.

8. Tsvetkov PO, Ezraty B, Mitchell JK, Devred F, Peyrot V, Derrick PJ, Barras F, Makarov AA, Lafitte D. Calorimetry and mass spectrometry study of oxidized calmodulin interaction with target and differential repair by methionine sulfoxide reductases. Biochimie. 2005; 87:473-80. https:// doi.org/10.1016/j.biochi.2004.11.020.

9. Petrushanko IY, Lobachev VM, Kononikhin AS, Makarov AA, Devred F, Kovacic H, Kubatiev AA, Tsvetkov PO. Oxidation of $\mathrm{Ca} 2+$-binding domain of NADPH oxidase 5 (NOX5): toward understanding the mechanism of inactivation of NOX5 by ROS. PloS One. 2016; 11:e0158726. https://doi. org/10.1371/journal.pone.0158726.

10. Privalov PL, Khechinashvili NN, Atanasov BP. Thermodynamic analysis of thermal transitions in globular proteins. I. Calorimetric study of ribotrypsinogen, ribonuclease and myoglobin. Biopolymers. 1971; 10:186590. https://doi.org/10.1002/bip.360101009.

11. Baladi S, Tsvetkov PO, Petrova TV, Takagi T, Sakamoto H, Lobachov VM, Makarov AA, Cox JA. Folding units in calcium vector protein of amphioxus: structural and functional properties of its amino- and carboxy-terminal halves. Protein Sci Publ Protein Soc. 2001; 10:771-8. https://doi.org/10.1110/ps.40601. 
12. Garbett NC, Mekmaysy CS, DeLeeuw L, Chaires JB. Clinical application of plasma thermograms. Utility, practical approaches and considerations. Methods. 2015; 76:41-50. https://doi.org/10.1016/j.ymeth.2014.10.030.

13. Garbett NC, Miller JJ, Jenson AB, Miller DM, Chaires JB. Interrogation of the plasma proteome with differential scanning calorimetry. Clin Chem. 2007; 53:2012-4.

14. Garbett NC, Mekmaysy CS, Helm CW, Jenson AB, Chaires JB. Differential scanning calorimetry of blood plasma for clinical diagnosis and monitoring. Exp Mol Pathol. 2009; 86:186-91. https://doi.org/10.1016/j.yexmp.2008.12.001.

15. Zapf I, Fekecs T, Ferencz A, Tizedes G, Pavlovics G, Kálmán E, Lrinczy D. DSC analysis of human plasma in breast cancer patients. Thermochim Acta. 2011; 524:88-91. https://doi.org/10.1016/j.tca.2011.06.019.

16. Todinova S, Krumova S, Gartcheva L, Robeerst C, Taneva SG. Microcalorimetry of blood serum proteome: a modified interaction network in the multiple myeloma case. Anal Chem. 2011; 83:7992-8. https://doi.org/10.1021/ac202055m.

17. Todinova S, Krumova S, Kurtev P, Dimitrov V, Djongov L, Dudunkov Z, Taneva SG. Calorimetry-based profiling of blood plasma from colorectal cancer patients. Biochim Biophys Acta. 2012; 1820:1879-85. https://doi. org/10.1016/j.bbagen.2012.08.001

18. Chagovetz AA, Jensen RL, Recht L, Glantz M, Chagovetz AM. Preliminary use of differential scanning calorimetry of cerebrospinal fluid for the diagnosis of glioblastoma multiforme. J Neurooncol. 2011; 105:499-506. https://doi. org/10.1007/s11060-011-0630-5.

19. Chagovetz AA, Quinn C, Damarse N, Hansen LD, Chagovetz AM, Jensen RL. Differential scanning calorimetry of gliomas: a new tool in brain cancer diagnostics? Neurosurgery. 2013; 73:289-295; discussion 295. https://doi.org/10.1227/01.neu.0000430296.23799.cd.

20. Wen PY, Macdonald DR, Reardon DA, Cloughesy TF, Sorensen AG, Galanis E, Degroot J, Wick W, Gilbert MR, Lassman AB, Tsien C, Mikkelsen T, Wong ET, et al. Updated response assessment criteria for high-grade gliomas: response assessment in neuro-oncology working group. J Clin Oncol. 2010; 28:1963-72. https://doi. org/10.1200/JCO.2009.26.3541.

21. Ternant D, Cézé N, Lecomte T, Degenne D, Duveau AC, Watier H, Dorval E, Paintaud G. An enzymelinked immunosorbent assay to study bevacizumab pharmacokinetics. Ther Drug Monit. 2010; 32:647-52. https://doi.org/10.1097/FTD.0b013e3181ef582a.

22. Roman AY, Devred F, Lobatchov VM, Makarov AA, Peyrot V, Kubatiev AA, Tsvetkov PO. Sequential binding of calcium ions to the B-repeat domain of $\mathrm{SdrD}$ from Staphylococcus aureus. Can J Microbiol. 2016; 62:123-9. https://doi.org/10.1139/cjm-2015-0580. 